\title{
Substitution von seltenerdhaltigen Primärrohstoffen durch in der Produktion anfallenden Polierschlamm
}

\author{
Klaus Philipp Sedlazeck • Georg Winkler · Susanne Hiden · Kerstin Pfandl · Therese Schwarz • Walter Hermann • \\ Roland Pomberger
}

Online publiziert: 5. September 2017

(c) Der/die Autor(en) 2017. Dieser Artikel ist eine Open-Access-Publikation.

Zusammenfassung Die vorliegende Arbeit befasst sich mit der Einstufung von Polierschlamm aus der Glasverarbeitung als „Nebenprodukt“ mit dem Ziel, eine weitere Verwendung des Materials durch die Firma D. Swarovski KG $\mathrm{zu}$ ermöglichen. Durch eine Wiederverwendung des Schlamms könnten importierte und käuflich erworbene Primärrohstoffe substituiert werden. Dieser Schlamm enthält große Mengen an Seltenerdoxiden und fällt während des Polierprozesses an.

Die durchgeführten Versuche zeigen, dass durch den Einsatz des Polierschlamms Polierwerkzeuge hergestellt werden können, die den Qualitätsanforderungen der Firma entsprechen. Aufgrund des hohen Werts der enthaltenen Seltenerdoxide wurde mit der Implementation der kontaminationsfreien Abscheidung aus dem Prozesskreislauf begonnen. Das so rückgewonnene Material wurde nicht mehr deponiert, sondern am Firmengelände gelagert und für eine weitere Verwendung vorbereitet. Um das Material im vollen Umfang wieder einsetzen zu können und somit einen ökologischen und ökonomischen Vorteil zu schaffen, war die Einstufung des Materials als „Nebenprodukt“ erforderlich. Vor der Implementation der kontaminationsfreien Abscheidung lag das Material mit anderen Stoffen vermischt vor und musste als gefährlicher

K. P. Sedlazeck, M.Sc. $(\bowtie) \cdot S$. Hiden • DI K. Pfandl · Mag. T. Schwarz, B.Sc. . Univ.-Prof. DI Dr. R. Pomberger

Lehrstuhl für

Abfallverwertungstechnik

und Abfallwirtschaft,

Montanuniversität Leoben,

Franz-Josef-Straße 18, 8700 Leoben, Österreich

philipp.sedlazeck@unileoben.ac.at

G. Winkler $\cdot$ W. Hermann

D. Swarovski KG,

Swarovskistraße 30, 6112 Wattens, Österreich
Abfall entsorgt werden. Die Neukategorisierung als "Nebenprodukt" wurde in Verhandlungen der D. Swarovski KG mit den zuständigen österreichischen Behörden auf Basis der in der Arbeit vorgestellten Argumente und Ergebnisse erreicht. Diese Änderung trat im Dezember 2013 in Kraft und die industrielle Produktion von Polierwerkzeugen aus den so rückgewonnenen Seltenerdoxiden wurde im Jahr 2015 erfolgreich realisiert.

Substituting primary REE material by accruing polishing sludge from the production process

Abstract This paper deals with the declaration of polishing sludge - accruing during glass processing - as a "byproduct" in order to permit a further treatment of the material by the company D. Swarovski KG. A reutilization of the material is intended as a substitute for imported and purchased primary raw material. This sludge contains high amounts of rare earth oxides which are used during the polishing process. Experiments, conducted during this investigation, reveal an acceptable quality of the polishing tools which were produced by reusing this accrued sludge.

After implementation of contamination-free separation of the sludge from the processing cycle, the material was stored on site due to the high value of the contained rare earth oxides, instead of being disposed of on a landfill as "hazardous waste" mixed with other wastes. The company was - and still is - not a certified waste processing company; therefore, a change of the classification from "hazardous waste" to "by-product" was required in order to be able to reuse the emerging material. This leads to both, ecologic and economic advantages. On the basis of the arguments and results presented in our paper, D. Swarovski KG was able to debate with the appropriate Austrian authorities and successfully reached a re-categorization as a "by-product". This argumentation was accepted in December 2013. The industrial production of polishing tools from the reused material started successfully in 2015.

Keywords Polishing sludge $\cdot$ Reuse Recycling · By-product · Rare earth elements $\cdot$ Rare earth oxides

\section{Einleitung}

Der vorliegende Artikel fasst ein durch die D. Swarovski KG initiiertes Projekt zusammen, welches den firmeninternen Wiedereinsatz von Polierschlamm als Ziel hatte und in Zusammenarbeit mit der Montanuniversität Leoben durchgeführt wurde. Die D. Swarovski KG mit Sitz in Wattens ist ein „Kristallglas“-Fertigungs- und -Verarbeitungsunternehmen mit Fokus auf mechanischer Glasbehandlung, insbesondere Oberflächenbehandlung. Während des Herstellungsprozesses wird Glas geschliffen und poliert. Durch mechanische Reibung tritt Abrieb auf, weshalb die Polierwerkzeuge verschleißen und von Zeit zu Zeit ausgetauscht werden müssen. In der Vergangenheit wurde der entstandene Polierschlamm, der hohe Mengen an Seltenerdoxiden (SEO) enthält, zusammen mit anderen im Prozess anfallenden Abfällen deponiert und weder wiederverwendet noch recycelt. Durch die Deponierung gingen folglich auch die in den Polierschlämmen enthaltenen SEO verloren. Nach einer Änderung des Produktionsprozesses konnte das Unternehmen den Polierschlamm kontaminationsfrei separieren und sammeln. Seit dieser Implementierung wird der Schlamm in Big-Bags auf dem Werksgelände der D. Swarovski KG gelagert, für die Produktion von Polierwerkzeugen vorbereitet und eingesetzt und ersetzt einen Teil der Primärrohstoffe.

Die Polier- und Schleifwerkzeuge werden in der hauseigenen Werkstatt produziert. Sie bestehen primär aus 
Lanthan- und Ceroxiden, welche zur Gruppe der Seltenen Erden (SE) gehören und als kritische Rohstoffe eingestuft sind. Die Oxide werden in eine Matrix eingebettet, die durch den Polierprozess aufgebraucht wird, während die Lanthan- und Ceroxide selbst weitgehend unverändert bleiben. Infolge der in Österreich gültigen Vorschriften, welche eine erforderliche Behandlung zur Wiederverwendung des Materials gesetzlich untersagten, musste der gesamte Bedarf an SEO von externen Herstellern zugekauft werden.

Im Jahr 2006 wurden im Unternehmen neue Abtrennaggregate installiert und es wurde mit der getrennten Sammlung von nicht kontaminiertem, gebrauchtem SE-Material aus Polierwerkzeugen begonnen. Die Sammlung und Zwischenlagerung bildeten die Basis für eine spätere Wiederverwendung, wodurch in weiterer Folge das Vorsorgeprinzip des österreichischen Abfallwirtschaftsgesetzes (AWG 2002) erfüllt werden kann. Darüber hinaus werden ökonomische Vorteile durch Kosteneinsparungen erreicht.

Im Jahr 2011 stammten fast 97 M-\% der Weltproduktion von SE aus China. Obwohl die Reserven weltweit gleichermaßen verteilt sind, besitzt China aufgrund seiner niedrigen Preise eine nahezu monopolistische Stellung am Weltmarkt. Eine willkürlich eingeführte Exportbeschränkung der chinesischen Regierung enthüllte die starke Abhängigkeit von diesem Hauptproduzenten und führte im Jahr 2011 zu explodierenden Weltmarktpreisen von SEO, die enorme Auswirkungen auf die industriellen SE-Importländer hatte (Humphries 2012). Erschwerend kommt hin$\mathrm{zu}$, dass der SE-Bergbau und die primäre Behandlung ernsthafte regionale Umweltprobleme verursacht (siehe Abschn. 3.4), welche nur durch die Entwicklung geeigneterer und nachhaltiger (Primär-)Produktions- und Verwertungsprozesse minimiert werden könnten.

Aus den genannten Gründen entstand der Bedarf der D. Swarovski KG nach einer weiteren Verarbeitung des aufkommenden Schlamms mit dem Ziel der Substitution importierter, primärer SEO in den Polierwerkzeugen. Für die Erlaubnis zur Wiederverwendung des anfallenden Schlamms, waren mehrere Fragen bezüglich der Klassifikation zu beantworten, z. B. ob es sich bei dem anfallenden Schlamm um ein „Nebenprodukt“ handelt oder nicht.
Um im vorliegenden Fall eine genaue Abgrenzung $\mathrm{zu}$ ermöglichen, müssen die Begriffe „Recycling“ und „Wiederverwendung" speziell für den anfallenden Schlamm diskutiert werden. Es gilt zu klären, ob der Schlamm und die daraus entstehenden Werkzeuge per Definition „wiederverwendet“ oder „recycelt" werden. „Wiederverwendung“ ist definiert als: "[...] jedes Verfahren, bei dem Erzeugnisse oder Bestandteile, die keine Abfälle sind, wieder für denselben Zweck verwendet werden, für den sie ursprünglich bestimmt waren;" und „Recycling“" ist: „[...] jedes Verwertungsverfahren, durch das Abfallmaterialien zu Erzeugnissen, Materialien oder Stoffen entweder für den ursprünglichen Zweck oder für andere Zwecke aufbereitet werden [...]" (Richtlinie 2008/98/EG). Nachfolgend wird erörtert, ob es sich beim anfallenden Schlamm um „Abfall“ oder ein „Nebenprodukt“ handelt.

\section{1 Österreichische Richtlinien}

Aufgrund der hohen Bleigehalte wurde der Schlamm als gefährlicher Abfall eingestuft (Abfallverzeichnisverordnung in BMLFUW 2004; Winkler et al. 2014) und musste deponiert werden, was zum Verlust der enthaltenen SEO führte. Die versuchsweise Aufbereitung der sortenrein gewonnenen Fraktion und die Fertigung von ersten WerkzeugPrototypen, welche zur Evaluation der firmeninternen Behandlungsmöglichkeiten durchgeführt wurden, erzielten bereits vielversprechende Ergebnisse. Um den vollständigen Wiedereinsatz des Materials rechtlich zu ermöglichen, musste zunächst der SEO-Schlamm als „Nebenprodukt" eingestuft werden.

Dem österreichischen Abfallwirtschaftsgesetz entsprechend (AWG 2002, $\S 2$ (3a)), das auf der europäischen Abfallrichtlinie beruht (Richtlinie 2008/98/EG, Kap. 1, Artikel 5), wird ein Material als „Nebenprodukt“ („Ein Stoff oder Gegenstand, der das Ergebnis eines Herstellungsverfahrens ist, dessen Hauptziel nicht die Herstellung dieses Stoffes oder Gegenstands ist [...]") eingestuft, wenn folgende Voraussetzungen erfüllt sind:

1. „es ist sicher, dass der Stoff oder Gegenstand weiterverwendet wird;

2. der Stoff oder Gegenstand kann direkt, ohne weitere Verarbeitung, die über die normalen industriellen Verfahren hinausgeht, verwendet werden;
3. der Stoff oder Gegenstand wird als integraler Bestandteil eines Herstellungsprozesses erzeugt und

4. die weitere Verwendung ist zulässig, insbesondere ist der Stoff oder Gegenstand unbedenklich für den beabsichtigten sinnvollen Zweck einsetzbar, es werden keine Schutzgüter (vergleiche $\$ 1$ Abs. 3) durch die Verwendung beeinträchtigt und es werden alle einschlägigen Rechtsvorschriften eingehalten.“

Um die Frage zu beantworten, ob der Polierschlamm als „Nebenprodukt“ eingestuft werden kann, wurde die Einhaltung der angeführten Kriterien geprüft, wie in den folgenden Kapiteln beschrieben.

\section{Material und Methoden}

\subsection{Swarovski KG}

Die Firma D. Swarovski KG befasst sich mit der mechanischen Bearbeitung von Glas zur Herstellung von Schmuck und Dekorationselementen. Ein Teil dieses Herstellungsprozesses ist das Polieren. Während dieses Vorgangs entsteht durch Abrieb von Polierwerkzeugen und dem polierten Glas ein Schlamm, der auch andere Bestandteile enthält, die während des Verfahrens verwendet werden.

Jedes Jahr wurden 150 bis 200 Tonnen SE im Zuge der Deponierung des Schlamms entsorgt (Winkler et al. 2014). Die Installation eines separaten Polierwasserkreislaufs inklusive einer Feststoffausschleusung im Jahr 2006 erlaubte eine getrennte Sammlung der wertvollen SEO-haltigen Polierschlämme sowie der Schleif- und Polierwässer. Diese Installation wurde im Jahr 2011 erfolgreich fertiggestellt. Seither wird der Schlamm vor Ort gelagert und nicht mehr deponiert. 2008 wurde eine Wiederverwendung durch die Verwendung von bleifreiem Glasmaterial sowie der chlorfreien Herstellung der Polierwerkzeuge zusätzlich erleichtert.

Der Aufbereitungszyklus, der die Wiederverwendung des Polierschlammes ermöglicht, ist in Abb. 1 dargestellt.

Während des Polierprozesses fällt ein Schlamm mit hohem Wassergehalt an. Nach dem Sammeln wird er für eine erste Wasserreduktion eingedickt (10 bis $15 \mathrm{M}-\%$ Trockensubstanz) und im Anschluss in eine Filterpresse eingebracht. Nach dem Pressvorgang erreicht das Material einen Trocken- 


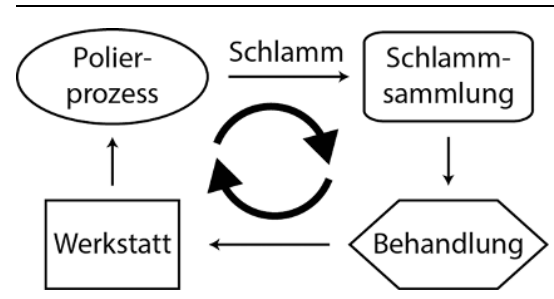

Abb. 1 Polierschlamm-Aufbereitungszyklus der D. Swarovski KG. Sämtliche Materialverluste aus den Prozessen werden in dieser schematischen Übersicht vernachlässigt

substanzgehalt von 75 bis $80 \mathrm{M}-\%$. Das durch die Filterpresse abgetrennte Prozesswasser wird auf die Anforderungen der Poliermaschinen konditioniert und in den internen Prozesswasserkreislauf rückgeführt. Bevor der entwässerte Schlamm in Big-Bags gefüllt und auf dem Fabrikgelände gelagert wird, wird er durch ein Zerkleinerungsaggregat (Nibbler) auf eine Korngröße von etwa $10 \mathrm{~mm}$ zerkleinert.

Um die Polierwerkzeuge in der hausinternen Werkstatt fertigen zu können, ist eine Korngröße von $<10 \mu \mathrm{m}$ und eine Trockensubstanz von annähernd $100 \mathrm{M}-\%$ erforderlich. Mit der entsprechenden Zerkleinerung und Trocknung wird ein externes Unternehmen beauftragt. Zusätzliche Behandlungsschritte zur Verbesserung der Qualität sind ebenfalls möglich. Im Moment gibt es aus wirtschaftlichen Gründen keine bestehenden Pläne für die Installation weiterer Behandlungsaggregate wie einer Mühle oder eines Trockners auf dem Fabrikgelände der D. Swarovski KG.

Nach der Rücklieferung des vermahlenen SEO-Materials vom externen Auftragnehmer werden die Polierwerkzeuge in der firmeneigenen Werkstatt gefertigt. Die Behandlung der sekundären Poliermittel erfolgt dabei gleich jener des Primärmaterials durch Pressen, Kneten, Gießen und Stampfen. Lediglich die Mischtanks, die für das Trockenmischen verwendet werden, sowie die Matrix der Scheiben variieren in Größe, Bauart und Eigenschaften entsprechend den aktuellen Anforderungen, da auf Anfrage verschiedene Arten von Polierwerkzeugen bedarfsorientiert hergestellt werden.

\subsubsection{Polierversuche}

Im Zuge der gegenständlichen Untersuchungen wurden vier verschiedene
Polierversuche mit unterschiedlichen Arten von Polierwerkzeugen durchgeführt. Zwei Werkzeuge (R1 und R2) wurden aus dem zurückgewonnenen SEO (wie oben beschrieben) hergestellt und zwei Werkzeuge (HQ und LQ) wurden aus zugekauftem Primärrohstoff hergestellt. Für das Werkzeug HQ wurde ein importierter hochwertiger Primärrohstoff, für das Werkzeug LQ einer mit geringerer Qualität eingesetzt.

Die Qualität der Werkzeuge, die aus verschiedenen Rohstoffen hergestellt wurden, wurde auf folgende Weise eruiert: Ein genormtes Glasstück wurde in die Glasschneidemaschine gespannt und 6 bis $7 \mathrm{~mm}$ der Probenoberfläche wurden entfernt. Danach wurde die verbleibende Probe entnommen, getrocknet und in die Ablationsmessvorrichtung gegeben. Die Ablationsmessvorrichtung wurde auf Null gesetzt und die Probe wieder in die Glasschneidmaschine gegeben. Vordefinierte Parameter wie Pendelhub der Maschine, Schnittgeschwindigkeit der Scheibe, Anpressdruck auf die Scheibe, Polierdauer, Zusammensetzung des Kühlschmiermittels, Temperatur- und Volumenstrom des Kühlschmiermittels, die Glaszusammensetzung und Probengeometrie sowie das Abrichten und Konditionieren der Polierscheiben sorgen für die Reproduzier- und Vergleichbarkeit der Polierprozesse. Nach dem Polieren wurde die Probe getrocknet, die Polierablation gemessen und mit jenen der anderen Scheiben verglichen.

\section{Ergebnisse und Diskussion}

Im folgenden Abschnitt wird die Frage, ob es sich beim anfallenden Polierschlamm um ein "Nebenprodukt“ oder „Abfall“ handelt, unter Berücksichtigung der österreichischen Gesetzgebung erörtert (vgl. Kriterien in Abschn. 1.1), diskutiert und beantwortet. Darüber hinaus werden die Ergebnisse der praktischen Versuche vorgestellt, die zeitgleich durchgeführt wurden.

\subsection{Ist es sicher, dass der Stoff oder} Gegenstand weiterverwendet wird?

Untersuchungen des anfallenden Schlamms zeigten, dass die beteiligten Lanthan- und Ceroxid-Körner während des Prozesses nicht verbraucht, sondern lediglich aus der Matrix in den Schlamm freigesetzt werden. Der abgetrennte Schlamm beinhaltet folglich die gleichen Materialien wie die verwende- ten Primärrohstoffe und kann daher als Substitution für Primärrohstoffe zur Herstellung der Polierwerkzeuge in der internen Werkstatt verwendet werden. Demnach ist eine Wiederverwendung des hochwertigen SE-haltigen Schlamms gewährleistet, da nach der Anpassung des Produktionszyklus eine sortenreine Abtrennung und Sammlung des Polierschlamms möglich ist.

Wie in Tab. 1 ersichtlich, verdeutlichen die in Abschn. 2.1.1 beschriebenen Versuche, dass mit Polierwerkzeugen, die aus zurückgewonnenen Material hergestellt wurden, gute Polierergebnisse erzielt werden können.

Mit einem Ablationsverhältnis von 65 bis $75 \%$ (normalisiert auf die Ergebnisse des HQ-Werkzeugs) ist die Effizienz der „Werkzeuge aus Sekundärrohstoff" nicht so hoch wie jene der Werkzeuge aus hochwertigem, zugekauftem Primärrohstoff (HQ). Allerdings waren die erzielten Ergebnisse mit den „Werkzeugen aus Sekundärrohstoff" deutlich besser als die Ergebnisse mit den Werkzeugen aus dem weniger hochwertigem, zugekauften Primärrohstoff (LQ), die ebenfalls als Standardwerkzeuge im Produktionsprozess zum Einsatz kommen. Die durchgeführten Versuche konnten verdeutlichen, dass der Lanthanoxid- und Ceroxid-Schlamm wiederverwendet werden kann, um geeignete Polierwerkzeuge für den Produktionsprozess zu herzustellen.

\subsection{Kann der Stoff oder Gegenstand} direkt ohne weitere Verarbeitung, die über die normalen industriellen Verfahren hinausgeht, verwendet werden?

Wie bereits beschrieben, müssen zusätzlich zum Aufbereitungszyklus weitere Schritte angewendet werden, um den anfallenden Schlamm in den $\mathrm{Zu}$ stand $\mathrm{zu}$ überführen, welcher für die Herstellung der Polierwerkzeuge erforderlich ist.

Diese zusätzlichen Schritte sind Trocknen, Pressen, Zerkleinern, Brechen und Mahlen. Nach der Definition von Schink et al. (2012) gehören diese Methoden zur Gruppe der üblichen industriellen Behandlung. Die Definition von Schink et al. (2012) impliziert, dass industrielle Prozesse alle Prozesse sind, die in der Primärproduktion verwendet werden und nicht ausschließlich in der Abfallbehandlung Anwendung finden. Obwohl die Grenzen dieser Definition nicht eindeutig sind, fallen die oben 
Tab. 1 Ergebnisse der Polier-Versuche unter Verwendung der verschiedenen Polierwerkzeuge

\begin{tabular}{|l|l|l|l|}
\hline Proben Nr & Werkzeug Name & Material & Ablation [\%] \\
\hline 1 & R1 & Zurückgewonnener Schlamm & 74 \\
\hline 2 & R2 & Zurückgewonnener Schlamm & 65 \\
\hline 3 & HQ & Hochwertiger zugekaufter Primärrohstoff & 100 \\
\hline 4 & LQ & Geringwertiger zugekaufter Primärrohstoff & 41 \\
\hline
\end{tabular}

beschriebenen Prozesse in die Kategorie der Standardbehandlungsprozesse, die bei jeder Primärproduktion zur Anwendung kommen.

Darüber hinaus haben Schink et al. (2012) erklärt, dass übliche industrielle Behandlungen Prozesse sind, die ein Produkt marktfähig machen, ohne seine Eigenschaften zu verändern. Als Beispiel kann das Mahlen von Schlacken als übliche industrielle Behandlung eingestuft werden. Ein Prozess jedoch, der unerwünschte Teile der Probe entfernt, würde deren Eigenschaften verändern und folglich fiele diese Entfernung nicht unter die industriellen Standardbehandlungsprozesse.

Jene Prozesse, die für die firmeninterne Wiederverwendung des Polierschlamms notwendig sind (Trocknen, Pressen, Zerkleinern und Mahlen), gehören zu den grundlegenden Prozessen in der Primärrohstoffindustrie, welche die Materialeigenschaften nicht verändern. Damit kann begründet werden, dass durch die Adaption des Aufbereitungszyklus keine Verarbeitung über die üblichen industriellen Behandlungsmethoden hinaus erfolgt.

\subsection{Wird der Stoff oder Gegenstand als integraler Bestandteil eines Herstellungsprozesses erzeugt?}

Wie bereits beschrieben, liegt das Hauptaugenmerk der D. Swarovski KG auf der Herstellung von „Kristallglas“ für Schmuck und Dekoration, und einer der Produktionsschritte ist das Polieren. Dabei werden neben der Glasoberfläche auch die Polierwerkzeuge abgetragen. Zum Kühlen, zur Staubreduktion und zum Abtransport des Abriebmaterials wird ein speziell konditioniertes Prozesswasser verwendet, welches abgetrennt und in den Prozesswasserkreislauf rückgeführt wird. Der dabei entstehende Schlamm ist ein wesentlicher integraler Bestandteil des Produktionsprozesses und fällt kontinuierlich an
3.4 Ist die weitere Verwendung zulässig und werden keine Schutzgüter durch die Verwendung beeinträchtigt?

Aus Polierschlamm hergestellte Polierwerkzeuge unterscheiden sich nicht von den aus Primärrohstoffen hergestellten Produkten (vgl. Abschn. 3.1). Da die Polierwerkzeuge aus dem Primärwerkstoff unter Berücksichtigung des beabsichtigten Verwendungszwecks zulässig und nicht gefährlich sind, erfüllen auch die aus dem Schlamm hergestellten Werkzeuge dieses Kriterium.

Um als „Nebenprodukt“ und nicht als „Abfall“ eingestuft zu werden, dürfen keine Schutzgüter beeinträchtigt werden. Laut AWG (2002) $§ 1$ (3) ist dies erfüllt, wenn die öffentlichen Interessen gewahrt bleiben. Um dies zu erfüllen, müssen folgende Kriterien ausgeschlossen werden:

- Gefährdung menschlicher Gesundheit,

- Gefährdung für Wasser, Luft, Boden, Tiere,

- Beeinträchtigung der nachhaltigen Nutzung von Wasser oder Boden,

- Umweltverschmutzung über das unvermeidliche Maß,

- Entstehung von Brand oder Explosionsgefahren,

- Entstehung übermäßigen Lärms,

- Auftreten oder Entstehung von Krankheitserregern,

- Störung der öffentlichen Ordnung und

- Beeinträchtigung der Landschaft oder von Kulturgütern.

Aufgrund der am Werksgelände bestehenden hohen Sicherheitsstandards kann verhindert werden, dass es bei der Wiederverwendung des anfallenden Schlamms zu einer Beeinträchtigung der öffentlichen Interessen kommt. Es ist kein Schutzgut betroffen und alle Vorschriften sind erfüllt.

Da die Verwendung der Werkzeuge, die aus zugekauftem Primärrohstoff hergestellt werden, beim Polieren keine Gefährdung für die Schutzziele darstellt, können die Polierwerkzeuge, welche aus den rückgewonnenen SEO hergestellt werden, ebenfalls keine Gefährdung darstellen. Diese bestehen aus einem Rohstoff ähnlicher Zusammensetzung und werden demselben Fertigungsprozess unterzogen.

Die am Standort ohnedies implementierten Umweltschutzmaßnahmen verhindern eine Beeinträchtigung öffentlicher Interessen. Die für die Herstellung des Originalwerkzeugs bereits installierten Luftfilteranlagen gewährleisten, dass keine zusätzlichen Umweltemissionen zu erwarten sind. Eine weitere Verwendung der rückgewonnenen Rohstoffe aus dem Polierschlamm verringert zudem die Menge an zu deponierendem gefährlichen Abfall, was zur Einsparung von Deponiekapazitäten führt. Die Verringerung der Masse, die deponiert werden muss, entspricht weitgehend der Masse des anfallenden Polierschlamms. Jüngste Zahlen zeigen einen deutlichen positiven Effekt.

Der Wiederverwertungskreislauf und seine Auswirkungen sind in Abb. 2 dargestellt. In der Vergangenheit wurde die gesamte Menge des anfallenden Schlamms deponiert. Durch die Wiederverwertung ist es folglich gelungen, in der im AWG (2002) $\S 1$ (2) verankerten Abfallhierarchie von Stufe 5, Beseitigung, auf Stufe 2, Wiederverwertung, zu klettern. Das vorliegende Projekt handelt somit zur Gänze im Sinne des Vorsorgeprinzips und der Nachhaltigkeit der Abfallwirtschaft.

2014 wurden erweiterte Versuche mit den Polierschlamm-Werkzeugen erfolgreich durchgeführt, jedoch fand $\mathrm{zu}$ diesem Zeitpunkt noch keine Wiederverwendung statt. Demnach kam es weder $\mathrm{zu}$ einer Verringerung der Primärrohstoffeinsatzes, noch zu einer Einsparung von Deponiekapazitäten.

Im Jahr 2015 wurden bereits $20 \%$ des zurückgewonnenen Schlamms wiederverwendet. Im Jahr 2016 war ein Anstieg auf $26 \%$ geplant, was die hohe Effizienz dieses implementierten Wiederverwendungsprozesses aufzeigt. Das langfristige Ziel ist es, 80 bis $90 \%$ des Primärrohstoffs zu ersetzen.

Außerdem trägt die Wiederverwendung des Lanthan- und Ceroxid enthaltenden Schlamms zum Schutz der Umwelt bei. Wie bereits beschrieben, werden 97 M-\% der SE der Welt in China abgebaut und aufbereitet. Abbau und Aufbereitung von SE in China haben eine ernsthafte regionale Umweltverschmutzung zur Folge. Da die gesamte, 


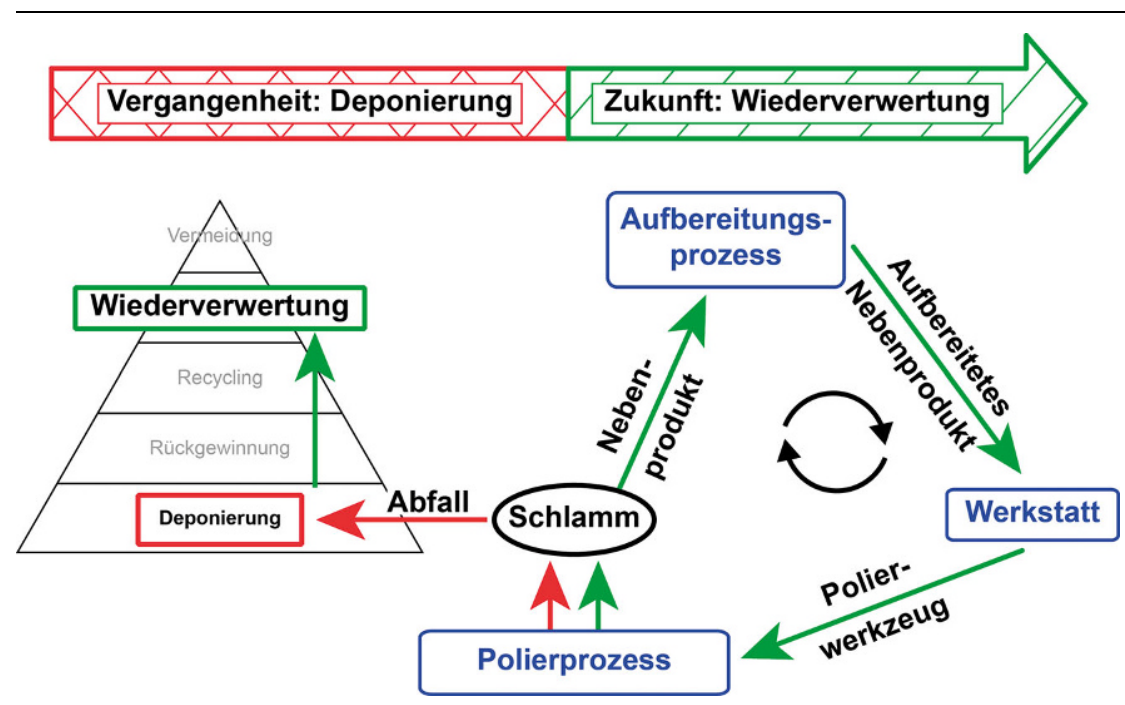

Abb. 2 Von der Deponierung zur Wiederverwertung des Polierschlamms der D. SwarovskiKG

für die Produktion der D. Swarovski KG verwendete Menge an SEO importiert werden muss, ist eine Substitution des importierten Primärrohstoffs durch den anfallenden Schlamm daher auch von großem ökologischen Interesse.

Bei der Herstellung von 100.000 Tonnen SE-Konzentrat wird eine geschätzte Menge von 200 Tonnen an radioaktivem Thoriumoxid erzeugt. Der bei der Produktion anfallende Schlamm enthält hohe Mengen an Schwefel- und Flusssäure sowie weitere Chemikalien, die zur Aufbereitung verwendet werden (Vateva 2012). Darüber hinaus sind die hohen Mengen an sauren Abwasserund Abgasemissionen in China problematisch, da die Stoffe weitgehend unbehandelt in die Umwelt entlassen werden. Das Abgas besteht typischerweise aus Staubkonzentrat, Fluorwasserstoffsäure, Schwefeldioxid und Schwefelsäure. Letztere werden durch die Verwendung konzentrierter Schwefelsäure während einer Hochtemperatur-Kalzinierungstechnik produziert (Hurst 2010).

Bei der Raffination einer Tonne SEO wird ein potenzielles Gasvolumen von ca. $60.000 \mathrm{~m}^{3}$ freigesetzt (Walters et al. 2010). Schueler et al. (2011) entdeckten, dass in der Umgebung eines chinesischen Produktionsstandortes Böden um den Faktor 1,7 und Pflanzen um den Faktor 32 höhere spezifische Radioaktivitäten aufwiesen. Li et al. (2013) berichteten von SE-Konzentrationen im Trinkwasser in der Region in der Nähe eines Bergbaugebietes, die 53 mal höher waren als im Vergleich zum Trinkwasser der Stadt. Casado-Martinez
(2013) zeigte, dass die Aufnahme von hohen SE-Konzentrationen toxisch für den menschlichen Organismus ist.

Diese Literaturdaten untermauern, dass ausgehend von der Primärproduktion und der Weiterverarbeitung von SE offensichtlich ein hohes Umweltverschmutzungspotenzial ausgeht. Eine Wiederverwendung des anfallenden Polierschlammes in der D. Swarovski KG führt zu einer Verringerung des Primärrohstoffeinsatzes zugunsten der Umwelt (kein Anfall von Thoriumoxid, kein Einsatz von Chemikalien, deutlich reduzierte Emissionen).

\subsection{Weitere Argumente für eine Wiederverwertung}

Ab 2009 stiegen Weltmarktpreise für Cer- und Lanthanoxide deutlich von rund 5 US \$/kg auf 150 US \$/kg im Jahr 2011, da China die Exportquoten beschränkte (Massari und Ruberti 2013). Ab 2011 sanken die Preise für Cer- und Lanthanoxide kontinuierlich bis auf 2 bis 2,5 US \$/kg im Jahr 2015 (BGR 2016). 2014 lagen die Preise unter dem Level von 2009, was mit der globalen wirtschaftlichen Rezession im Zusammenhang steht (Exner et al. 2016).

Seit 1999 regelte das chinesische Handelsministerium den Export von SE, und zwischen 2006 und 2010 wurden die Exportquoten weiter eingeschränkt. Im Jahr 2010 gab China bekannt, dass der Export aufgrund von Umweltschutzzielen und Einsparungen der Rohstoffbestände noch weiter beschränkt werden würde (Exner et al. 2016).
Die hohen Weltmarktpreise führten $\mathrm{zu}$ einer Kostensteigerung, die einen großen Einfluss auf die Wettbewerbsfähigkeit der Unternehmen in der westlichen Welt hatte. Im Jahr 2009 erhoben Kanada, USA, Mexiko, die Türkei und Österreich Einspruch gegen die Monopolstellung Chinas bei der Welthandelsorganisation. Darüber hinaus reichten die EU, Japan, Kanada und die USA im Jahr 2012 eine Beschwerde über die Exportquoten und die Preispolitik für SE ein. In beiden Fällen hat die Welthandelsorganisation zugunsten der Kläger entschieden. Das Argument war, dass die verfolgte Exportpolitik industriellpolitische Ziele verfolgt, anstatt Einsparungen von Rohstoffbeständen sowie Schutz der Umwelt (Exner et al. 2016).

Nach Graedel et al. (2011) betragen die durchschnittlichen globalen Endof-Life-Recyclingraten für Cer und Lanthan weniger als $1 \%$. Recyclingprozesse für SE sind komplex und verursachen einen entsprechend hohen Energieverbrauch (Gunn 2014), sodass ein Recycling bzw. eine Wiederverwendung unter Betrachtung der derzeit relativ niedrigen Weltmarktpreise - oftmals nicht in Relation zu den entstehenden Kosten steht. Trotz der sinkenden Weltmarktpreise ist die Produktion der Polierwerkzeuge aus dem anfallenden Schlamm nach wie vor wirtschaftlich rentabel und bringt zusätzlich Unabhängigkeit von Weltmarktpreisschwankungen. Darüber hinaus werden Entsorgungskosten eingespart.

Vorteile aus Sicht der Umwelt sind, dass für den Produktionsprozess weniger SE aufbereitet werden müssen, was die beschriebenen negativen Auswirkungen auf die Umwelt reduziert (u. a. radioaktive und staubförmige Emissionen). Zusätzlich werden Deponieressourcen geschont.

Laut Gunn (2014) ist die durch Wiederverwendung hervorgerufene Qualitätsminderung der größte Nachteil. Zudem ändert sich Produktdesign schnell mit zunehmendem technologischen Wissen. Die Qualität des Sekundärrohstoffs aus Polierschlamm ist tatsächlich niedriger als die des Primärrohstoffs höchster Qualität, jedoch ist die Qualität der Polierwerkzeuge aus Polierschlamm nach wie vor höher als jene von Werkzeugen aus Rohstoffen niedriger Qualität, welche ebenfalls als Standardwerkzeuge für den Produktionsprozess im Einsatz sind.

Zumal sich die Herstellung von Polierwerkzeugen aus dem wiederver- 
wendeten Schlamm direkt aus dem bereits bestehenden Produktionsprozess der Werkzeuge aus Primärrohstoffen ergibt, können Änderungen des Produktdesigns des Poliermittels ebenfalls problemlos übernommen werden. Hinzu kommt, dass sich die Zusammensetzung des Produktionsabfalls (neu anfallender Polierschlamm) mit dem eingesetzten Poliermittel während der Produktion ändert und somit der Wiederverwendungsprozess automatisch mitangepasst werden kann. Große Änderungen des Produktdesigns sind jedoch nicht zu erwarten.

\section{Schlussfolgerung}

Um den SE-Schlamm aus dem Polierprozess als „Nebenprodukt“ einstufen zu können, musste die Erfüllung der vier im österreichischen Abfallwirtschaftsgesetz (AWG 2002) $§ 2$ (3a) festgelegten Bedingungen nachgewiesen werden. Fragen und Antworten werden an dieser Stelle zusammengefasst.

- Ist es sicher, dass der Stoff oder Gegenstand weiterverwendet wird? Eine direkte Verwendung des Polierschlamms ist möglich und eine weitere Verwendung des Schlamms ist gewährleistet.

- Kann der Stoff oder Gegenstand direkt ohne weitere Verarbeitung, die über die normalen industriellen Verfahren hinausgeht, verwendet werden?

Es ist zwar eine weitere Behandlung erforderlich, aber es wird gezeigt, dass die dazu notwendigen Prozesse in die Kategorie der Standardbehandlungs- prozesse fallen, die bei jeder Primärproduktion Anwendung finden und damit zu den üblichen industriellen Prozessen zählen.

- Wird der Stoff oder Gegenstand als integraler Bestandteil eines Herstellungsprozesses erzeugt?

Das Hauptziel der D. Swarovski KG ist es, „Kristallglas“ für Schmuck und für die Dekoration zu produzieren. Der anfallende Schlamm entsteht als integrierter Bestandteil eines Produktionsprozesses.

- Ist die weitere Verwendung zulässig und werden keine Schutzgüter durch die Verwendung beeinträchtigt? Es wurde bewiesen, dass das Produkt aus Polierschlamm keine negativen Auswirkungen auf die Umwelt hat. Das genaue Gegenteil ist der Fall: Durch die Wiederverwendung des anfallenden Schlamms fallen im Gegensatz zur Primärrohstofferzeugung keine vergleichbaren oder bedenklichen Umweltverschmutzungen an. Hinzu kommt, dass die zu deponierende Abfallmenge reduziert wird.

Darüber hinaus ergibt sich durch die Substitution von primärem SEO in den Polierwerkzeugen durch die Wiederverwertung des Schlamms eine wirtschaftlicher Vorteil für die D. Swarovski KG.

Auf Grundlage der vorgelegten Forschungsergebnisse und der Argumentation haben die österreichischen Behörden Ende 2013 entschieden, dass - unter Berücksichtigung des österreichischen Abfallwirtschaftsgesetzes der anfallende Schlamm ein „Nebenprodukt“ und kein „Abfall“ ist. Unter strengen Bedingungen ist es dem Unternehmen erlaubt, die notwendigen Behandlungsschritte durchzuführen um das Material für die Herstellung neuer Polierwerkzeuge wiederzuverwenden. Jüngste Zahlen bestätigen die Eignung dieses innovativen Prozesses, da bereits im Jahr $201520 \%$ des anfallenden Schlamms wiederverwendet wurden. Das langfristige Ziel ist es, 80 bis $90 \%$ des Primärrohstoffs zu substituieren.

Danksagung Die Autoren bedanken sich bei Dr. Martin Eisenberger für rechtliche Unterstützung bei der Kommunikation mit den österreichischen Behörden sowie für das Sammeln von Informationen und Daten. Der D. Swarovski KG danken wir für die gute Zusammenarbeit in diesem Projekt.

Funding Open access funding provided by Montanuniversität Leoben.

Open Access Dieser Artikel wird unter der Creative Commons Namensnennung 4.0 International Lizenz (http:// creativecommons.org/licenses/by/4. $0 /$ deed.de) veröffentlicht, welche die Nutzung, Vervielfältigung, Bearbeitung, Verbreitung und Wiedergabe in jeglichem Medium und Format erlaubt, sofern Sie den/die ursprünglichen $\mathrm{Au}$ tor(en) und die Quelle ordnungsgemäß nennen, einen Link zur Creative Commons Lizenz beifügen und angeben, ob Änderungen vorgenommen wurden.
Abfallwirtschaftsgesetz (AWG) (2002): Bundesgesetz über eine nachhaltige Abfallwirtschaft (Abfallwirtschaftsgesetz 2002 - AWG 2002) StF: BGBl. I Nr. 102/

Bundesanstalt für Geowissenschaften und Rohstoffe (BGR) (2016): Preismonitor Januar 2016. DERA - Deutsche Rohstoffagentur.

Bundesministerium für Land- und Forstwirtschaft, Umwelt und Wasserwirtschaft (BMLFUW) (2004): Verordnung des Bundesministers für Land- und Forstwirtschaft, Umwelt und Wasserwirtschaft, mit der die Abfallverzeichnisverordnung geändert wird.

Casado-Martinez, C. (2013): Ökotoxizität der Seltenen Erden - Infoblatt (In German).

Exner, A., Held, M., Kümmerer, K. (2016): Kritische Metalle in der Großen Transformation, Berlin Heidelberg, Springer.

Graedel, T.E., Allwood, J., Birat, J.P., Buchert, M., Hagelüken, C., Reck, B.K., Sibley, S.F., Sonnemann, G. (2011): What Do We Know About Metal Recycling Rates? Journal of Industrial Ecology 15: 355-366.
Gunn, G. (2014): Critical metals handbook, led. Chichester: Wiley.

Humphries, M. (2012): Rare Earth Elements: The Global Supply Chain, Congressional Research Service 7-5700; R41347.

Hurst, C. (2010): for Institute for the Analysis of Global Security (IAGS). China's Rare Earth Elements Industry: What Can the West Learn?.

Li, X., Chen, Z., Chen, Z., Zhang, Y. (2013): A human health risk assessment of rare earth elements in soil and vegetables from a mining area in Fujian Province, Southeast China. Chemosphere 93: 1240-1246.

Massari, S., Ruberti, M. (2013): Rare earth elements as critical raw materials: Focus on in ternational markets and future strategies. Resources Policy 38:36-43.

Richtlinie 2008/98/EG (2008): Richtlinie 2008/ 98/EG des Europäischen Parlaments und des Rates vom 19. November 2008 über Abfälle und zur Aufhebung bestimmter Richtlinien. Richtlinie 2008/98/EG.
Schink, A., Frenz, W., Queitsch, P. (2012): Das neue Kreislaufwirtschaftsgesetz 2012. Heidelberg; Hamburg: Rehm Verl.-Gruppe Hüthig Jehle Rehm.

Schueler, D., Buchert, M., Liu, R., Dittrich, S., Merz, C. (2011): for Öko-Institut e.V.. Study on Rare Earths and Their Recycling, Final Report for The Greens/EFA Group in the European Parliament; Darmstadt.

Vateva, A.( 2012): for Unabhängiges Institut für Umweltfragen e.V.. China's Rare-Earth Elements Policy and its Implications for Germany, Japan and the USA; Berlin.

Walters, A., Lusty, P., Chetwyn, C., Hill, A (2010): for British Geological Survey. Rare Earth Elements. Keyworth, Nottingham: www.MineralsUK.com.

Winkler, G., Hiden, S., Schwaninger, R., Sedlazeck, K.P., Pomberger, R. (2014): Kreislaufführung von SEE-haltigen Polierschlämmen. In: R. Pomberger et al. (Hrsg.) RecyDepoTech 2016. Tagungsband, Leoben, 8-11 November, pp. 583588. Lavanttal, Österreich: Christian Theiss. 